\title{
In Vivo Release of Vancomycin from Calcium Phosphate Cement
}

\author{
Kentaro Uchida $\mathbb{D}^{1},{ }^{1}$ Ken Sugo $\mathbb{D},{ }^{2}$ Takehiko Nakajima, ${ }^{2}$ Mitsufumi Nakawaki, ${ }^{1}$ \\ Shotaro Takano, ${ }^{1}$ Naoshige Nagura, ${ }^{1}$ Masashi Takaso, ${ }^{1}$ and Ken Urabe ${ }^{3}$ \\ ${ }^{1}$ Department of Orthopedic Surgery, Kitasato University School of Medicine, 1-15-1 Kitasato, Minami, Sagamihara, \\ Kanagawa 252-0375, Japan \\ ${ }^{2}$ Research and Development Department, HOYA Technosurgical Corporation, 1-1-110 Tsutsujigaoka, Akishima, Tokyo 196-0012, Japan \\ ${ }^{3}$ Department of Orthopedic Surgery, Kitasato University Medical Center, 6-100 Arai, Kitamoto, Saitama 364-8501, Japan
}

Correspondence should be addressed to Ken Sugo; ken.sugo@pc.hoya.co.jp

Received 26 February 2018; Revised 10 April 2018; Accepted 16 April 2018; Published 15 May 2018

Academic Editor: Abdelwahab Omri

Copyright (C) 2018 Kentaro Uchida et al. This is an open access article distributed under the Creative Commons Attribution License, which permits unrestricted use, distribution, and reproduction in any medium, provided the original work is properly cited.

\begin{abstract}
Calcium phosphate cement (CPC) has good release efficiency and has therefore been used as a drug delivery system for postoperative infection. The release profile of CPC has mainly been evaluated by in vitro studies, which are carried out by immersing test specimens in a relatively large amount of solvent. However, it remains unclear whether antibiotic-impregnated CPC has sufficient clinical effects and release in vivo. We examined the in vivo release profile of CPC impregnated with vancomycin (VCM) and compared this with that of polymethylmethacrylate (PMMA) cement. To evaluate the release profile in vitro, the test specimens were immersed in $10 \mathrm{~mL}$ sterile phosphate-buffered saline per gram of test specimen and incubated at $37^{\circ} \mathrm{C}$ for 56 days in triplicate. For in vivo experiments, the test specimens were implanted between the fascia and muscle of the femur of rats. Residual VCM was extracted from the removed test specimens to determine the amount of VCM released into rat tissues. CPC released more VCM over a longer duration than PMMA in vitro. Released levels of VCM from CPC/VCM in vivo were 3.4-fold, 5.0-fold, and 8.6-fold greater on days 1,7 , and 28 , respectively, than those released on the corresponding days from PMMA/VCM and were drastically greater on day 56 due to inefficient release from PMMA/VCM. The amount of VCM released from CPC and PMMA was much higher than the minimum inhibitory concentration $(1.56 \mu \mathrm{g})$ and lower than the detection limit, respectively. Our findings suggest that $\mathrm{CPC}$ is a suitable material for releasing antibiotics for local action against established postoperative infection.
\end{abstract}

\section{Introduction}

Periprosthetic joint infection after total joint arthroplasty is a serious complication that requires prompt treatment. The two-stage exchange procedure is an effective treatment option for such infections [1]. Intravenously administered antibiotics, such as vancomycin (VCM), have poor tissue transferability depending on the site of infection, which decreases their therapeutic potential [2]. The therapeutic potential of VCM can be improved by combining it with a carrier to increase its retention at infection sites in the first stage of the procedure [3].

Calcium phosphate cement (CPC) has good release efficiency and has therefore been used as a drug delivery system for postoperative infection. CPC can release a greater volume of antibiotic over a longer duration than polymethylmethacrylate (PMMA) cement [4-7], a finding that has also been confirmed clinically [8-10]. Antibiotic release from the cement material is triggered when external solvent penetrates the material's pores, causing the antibiotic to diffuse out of the material $[11,12]$. In vitro studies are carried out by immersing test specimens in a relatively large amount of solvent, thereby providing ideal conditions for antibiotic release. However, it is unclear whether such release also occurs in vivo because tissue fluid is expected to only fill a few implanted sites compared to in vitro studies in the field of orthopedic surgery.

Here, we implanted CPC impregnated with VCM into rat tissue between the fascia and muscle and evaluated the release profile in vivo. We also compared the release profiles in vitro. 


\section{Materials and Methods}

2.1. Materials. CPC (Biopex-R Advance) and PMMA (Surgical Simplex P Bone Cement) were obtained from HOYA Technosurgical (Tokyo, Japan) and Stryker (Tokyo, Japan), respectively. Injectable VCM hydrochloride was purchased from Shionogi (Osaka, Japan). All other chemicals were obtained from Wako Pure Chemical Industries (Osaka, Japan).

2.2. Preparation of Test Specimens. All preparations were performed aseptically. CPC powder (24 g), VCM ( $2 \mathrm{~g})$, and a dedicated solvent $(5.6 \mathrm{~mL})$ were uniformly mixed to make a paste before adding a further $1.6 \mathrm{~mL}$ of solvent for effective handling (final volume $7.2 \mathrm{~mL}$ ). The amount of VCM used $(2 \mathrm{~g})$ was the same as that used by our clinical team. The paste was applied to a silicone sheet containing 60 molds $(\varphi$ $10 \mathrm{~mm} \times t 2 \mathrm{~mm}$ ) and was hardened by incubating for $3 \mathrm{~h}$ at room temperature. For PMMA, polymer powder (24 g), VCM (2 g), and liquid monomer $(12 \mathrm{~mL})$ were uniformly mixed to make a paste and hardened in the same manner as for CPC. Once hardened, the molds were removed to obtain the test specimens, which were designated CPC/VCM and PMMA/VCM (Figure 1). The 60 total CPC/VCM and PMMA/VCM specimens were used as follows: 50 for in vivo testing, 3 for in vitro testing, and the remaining for morphological observation.

2.3. In Vitro Study. The test specimens were immersed in $10 \mathrm{~mL}$ sterile phosphate-buffered saline (PBS(-)) per gram of test specimen and incubated at $37^{\circ} \mathrm{C}$ for 56 days in triplicate. PBS(-) was replaced daily. Eluates were collected on days 1 , 7,28 , and 56 ( $n=3$ each). VCM was detected by highperformance liquid chromatography (HPLC) on the day of collection.

2.4. Determination of VCM Concentration. A series of standard solutions of differing known concentrations of VCM in PBS(-) were prepared and injected into a CAPCELLPAK C18 UG120 column $(5 \mu \mathrm{m}, \varphi 4.6 \mathrm{~mm} \times h 250 \mathrm{~mm}$; Shiseido, Tokyo, Japan) of an Elite LaChrom HPLC system (Hitachi High-Technologies, Tokyo, Japan) equipped with a L-2455 Diode Array Detector (Hitachi High-Technologies). The HPLC conditions were as follows: column temperature, $30^{\circ} \mathrm{C}$; mobile phase A, triethylamine buffer ( $\mathrm{pH} 3.2$ )/acetonitrile/tetrahydrofuran $=92 / 7 / 1(\mathrm{v} / \mathrm{v})$; isocratic elution, phase A $(20 \mathrm{~min})$; flow rate, $1 \mathrm{~mL} / \mathrm{min}$; wavelength, $280 \mathrm{~nm}$; and injection volume, $20 \mu \mathrm{L}$. The peak area of VCM in each standard solution was measured and plotted against the VCM concentration to generate a calibration curve. The concentration of VCM in each eluate sample was then determined by HPLC using the same conditions as those used to generate the standard calibration curve.

2.5. In Vivo Study. In vivo studies were approved by the Kitasato University School of Medicine and Hospital Ethics Committee (Approval number 2017-098). Wistar rats, 10

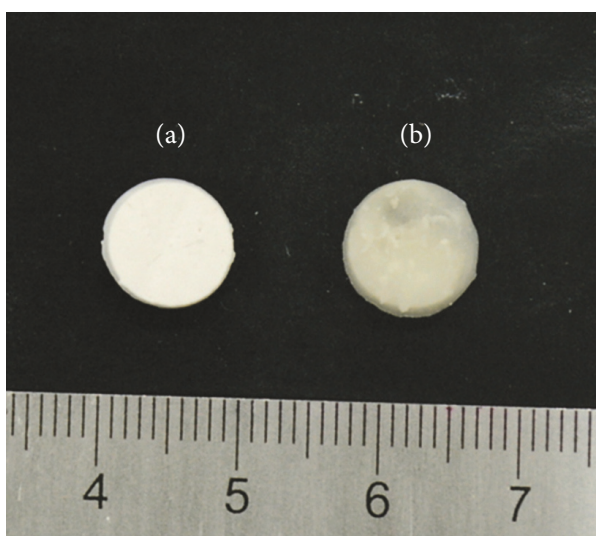

FIgURE 1: Photo of test specimens prepared at $\varphi 10 \mathrm{~mm} \times t$ $2 \mathrm{~mm}$. Calcium phosphate cement/vancomycin (CPC/VCM) (a) and polymethylmethacrylate (PMMA)/VCM (b).

weeks old, were anesthetized with $0.3 \mathrm{mg} / \mathrm{kg}$ medetomidine (Domitor, Nippon Zenyaku Kogyo, Fukushima, Japan), $0.5 \mathrm{mg} / \mathrm{kg}$ butorphanol (Vetorphale, Meiji Seika Pharma, Tokyo, Japan), and $0.5 \mathrm{mg} / \mathrm{kg}$ midazolam (Midazolam Sandoz, Sandoz, Tokyo, Japan). CPC/VCM and PMMA/VCM were implanted between the fascia and the muscle of the femur on the left and right side of 40 rats, respectively. The fascia and skin were sutured and the animals were allowed to move freely in their cages immediately after the surgery. Test specimens were removed on days $1,7,28$, and 56 , after sacrificing the animals ( $n=10$ each).

2.6. Extraction of VCM. Residual VCM was extracted from the removed test specimens on days $1,7,28$, and 56 to determine the amount of VCM released into rat tissues. CPC/VCM was removed and ground in a mortar, and $10 \mathrm{mg}$ of the powder was completely dissolved in $0.3 \mathrm{~mL}$ of $1 \mathrm{M}$ hydrochloric acid, followed by $2.7 \mathrm{~mL}$ of water. The solution was subsequently diluted 10 -fold with chromatographic mobile phase solution. PMMA/VCM was removed and crushed using an osteotome, and $10 \mathrm{mg}$ pieces were completely dissolved in $3 \mathrm{~mL}$ of acetone. The solution was sonicated for $1 \mathrm{~min}$ (UR-20P, Tomy Seiko, Tokyo, Japan), diluted 10 -fold with chromatographic mobile phase solution, and centrifuged at $5,000 \times \mathrm{g}$ for $5 \mathrm{~min}$ at $25^{\circ} \mathrm{C}(3700, \mathrm{Kub}-$ ota, Tokyo, Japan) to remove precipitates. Ten of each test specimen without implantation were used as a reference with $0 \%$ release. All extracted VCM was analyzed using HPLC as described above.

2.7. Morphological Observation. The relationship between the pore structure and VCM release profiles of CPC/VCM and PMMA/VCM was analyzed and compared. CPC/VCM without implantation was immersed in $20 \mathrm{~mL}$ of acetone for $10 \mathrm{~min}$ for dehydration, removed, and dried at room temperature; this process was not necessary for PMMA/VCM. Thin sections (1-2 mm thickness) of each test specimen were prepared using a microtome for scanning electron microscopy (SEM) analysis (S-4300, Hitachi High-Technologies) and the 


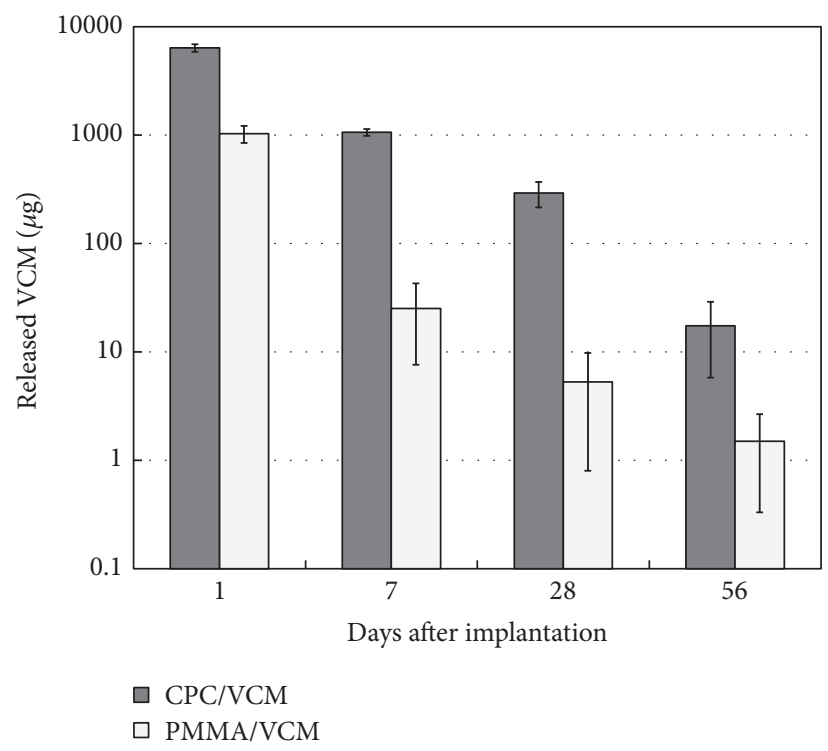

FIGURE 2: In vitro release of VCM from CPC/VCM and PMMA/VCM per day. Solvent was replaced daily and VCM levels were examined on days 1, 7, 28, and 56 by high-performance liquid chromatography. The average value and standard deviation in triplicate are shown.

analysis of pore size distribution using mercury porosimetry (AutoPore IV9520, Micromeritics, Norcross, GA, USA).

2.8. Statistical Analysis. The VCM release profile and the total amount of VCM released by CPC/VCM and PMMA/VCM were statistically compared using a $t$-test following a Levene test for equality of variance result of $p \geq 0.05$. Differences were considered significant if $p$ values were less than 0.05 .

\section{Results}

3.1. In Vitro Study. The amount of VCM released from CPC/VCM was greater than that from PMMA/VCM throughout the test period (Figure 2).

3.2. In Vivo Study. The amount of VCM released into rat tissues $\left(W_{t}\right)$ was calculated by subtracting the amount of VCM extracted from the removed test specimens from that extracted from the reference specimens without implantation. The symbol $t(=1,7,28$, and 56) indicates the number of days after implantation. Figure 3 shows $W_{1}, W_{7}, W_{28}$, and $W_{56}$ for CPC/VCM and PMMA/VCM, which represent the average amount of VCM released from 10 specimens removed on each day. CPC/VCM released significantly more VCM over a longer duration than PMMA in vivo, as was observed in vitro ( $p<0.001$, for all measured time points).

Similar to the in vitro studies described above, we examined the amount of VCM released per day instead of accumulated release. We generated an approximate curve from $W_{1}, W_{7}, W_{28}$, and $W_{56}$ (Figure 3 ) and calculated " $W_{t}-W_{t-1}$ " using the curve to determine the amount of VCM released per day. The amount of VCM released on days $1,7,28$, and

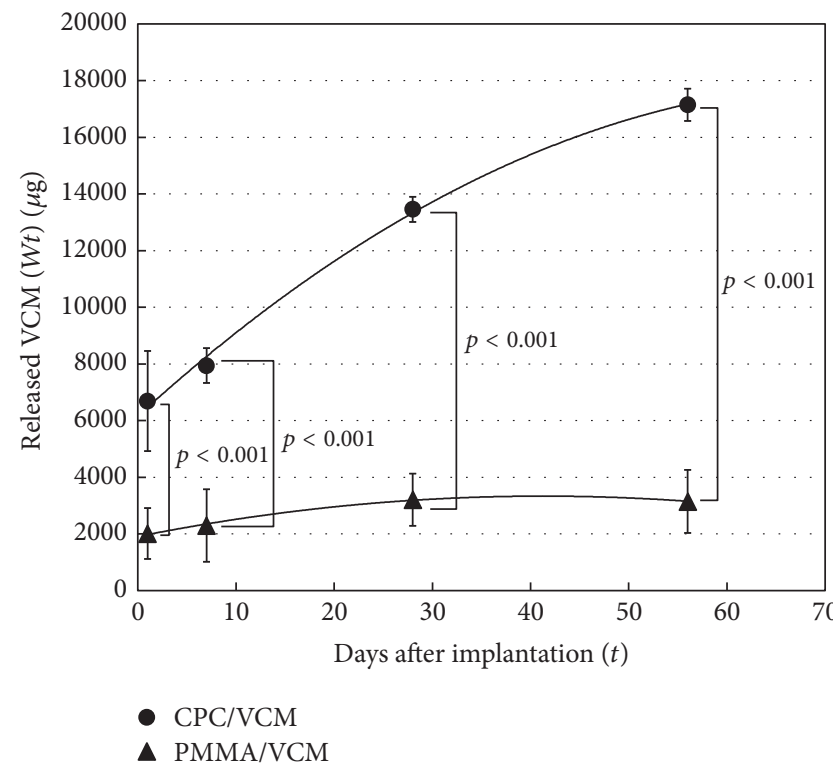

FIGURE 3: In vivo accumulated release of VCM from CPC/VCM and PMMA/VCM. Test specimens were removed from rats on days $1,7,28$, and 56, and the amount of VCM released into rat tissues was examined. Horizontal axis " $t$ " and vertical axis " $W_{t}$ " indicate the number of days after implantation and the amount of VCM released from each test specimen, respectively. The average value and standard deviation of 10 of each specimen are shown as $W_{1}, W_{7}, W_{28}$, and $W_{56}$.

56 is shown in Figure 4 . In contrast to $W_{1}, W_{7}, W_{28}$, and $W_{56}$ values, which were calculated as the average of 10 data points, $W_{t-1}$ was calculated from the approximate curve as one data point; there are therefore no error bars for data shown in Figure 4 . The release pattern of VCM from CPC/VCM into rat tissues was similar to that observed in the in vitro study. The mean amount of VCM released on day $56(79.8 \mu \mathrm{g})$ was much higher than the minimum inhibitory concentration (MIC) of $1.56 \mu \mathrm{g}$ per milliliter of local body fluid [13] for methicillinresistant Staphylococcus aureus. While the release pattern of VCM from PMMA/VCM was also similar to that observed in vitro, the mean amount of VCM released on day $56(<0.1 \mu \mathrm{g})$ was lower than the detection limit and the MIC. Released levels of VCM from CPC/VCM were 3.4-fold, 5.0-fold, and 8.6 -fold greater on days 1,7 , and 28 , respectively, than those released on the corresponding days from PMMA/VCM and were drastically greater on day 56 due to inefficient release from PMMA/VCM.

3.3. Comparison of Total Amount Released. Figure 5 shows the total amount of VCM released from CPC/VCM and PMMA/VCM into rat tissues in 56 days. The values for the in vivo study are indicated by the data on day 56 in Figure 3 $\left(W_{56}\right)$ and those for the in vitro study were calculated by summing values from the elution curves generated following the daily exchange of solvent. The total amount of VCM released by CPC/VCM in vivo was approximately half that released in vitro, at elution rates of $64.1 \%$ and $119.3 \%(p<0.001)$, respectively. Almost all impregnated VCM was released from 


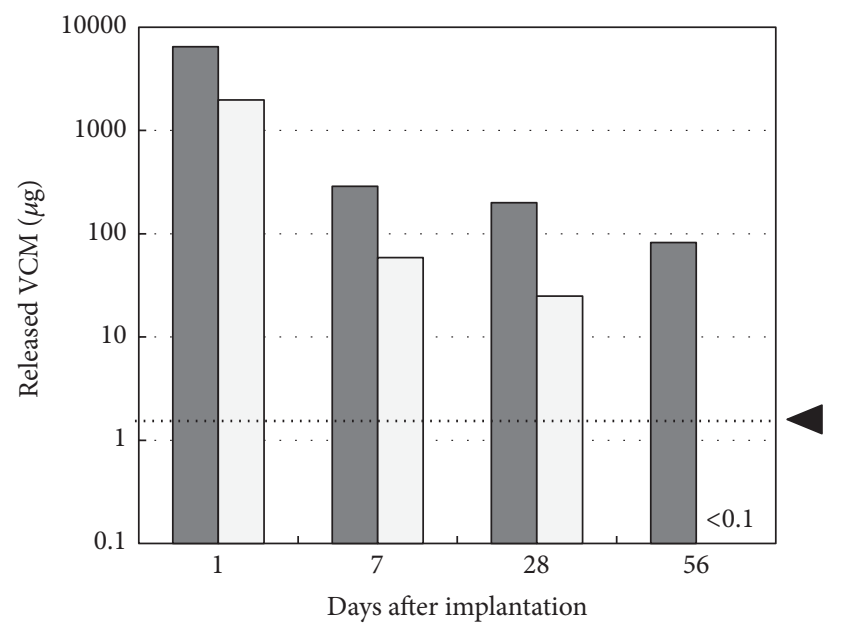

$\square \mathrm{CPC} / \mathrm{VCM}$

$\square \mathrm{PMMA} / \mathrm{VCM}$

FIGURE 4: In vivo release of VCM from CPC/VCM and PMMA/VCM per day. " $W_{t}-W_{t-1}$ " values were calculated from the approximate curve in Figure 3 . The closed arrowhead indicates the minimum inhibitory concentration $(1.56 \mu \mathrm{g})$.

$\mathrm{CPC} / \mathrm{VCM}$ in vitro, and our approximate curve suggests that $\mathrm{CPC} / \mathrm{VCM}$ has the potential to continue to release residual VCM fractions after day 56 in vivo. In contrast, the total amount of VCM released by PMMA/VCM in vivo was 2-fold greater than that released in vitro, at elution rates of $29.6 \%$ and $14.8 \%(p=0.046)$, respectively. The total amount of VCM released in vivo from CPC/VCM was 5.5-fold greater than that released from PMMA/VCM $(p<0.001)$.

3.4. SEM Observation and Porosimetry Analysis. SEM observation showed numerous pores on the surface of CPC/VCM (Figure 6(a)), which are formed by crystal growth and entanglement of $\alpha$-tricalcium phosphate particles [14]. In contrast, little to no pores were observed on the surface of PMMA/VCM (Figure 6(b)), which is formed by radical polymerization and is fundamentally different from the composition of CPC/VCM. Consistent with the SEM analysis, mercury porosimetry revealed that the test specimens had markedly different pore size distributions, with mode values of $0.22 \mu \mathrm{m}$ and $0.011 \mu \mathrm{m}$ for CPC/VCM and PMMA/VCM, respectively (Figure 7 ).

\section{Discussion}

Previous studies have reported that antibiotic-loaded bone cement spacers are useful for antibiotic release and inhibiting bacterial growth $[7,15,16]$. PMMA cement has long been proposed to permit release of impregnated antibiotics in vitro and in vivo $[16,17]$ and is still used as a standard antibacterial spacer, especially in mechanical strength-required sites [7]. The poor release of antibiotics from PMMA compared to CPC in vitro has been reported [4-7]. However, to date, the release profile of these materials has not been determined in vivo. Here, PMMA/VCM showed 2-fold greater release

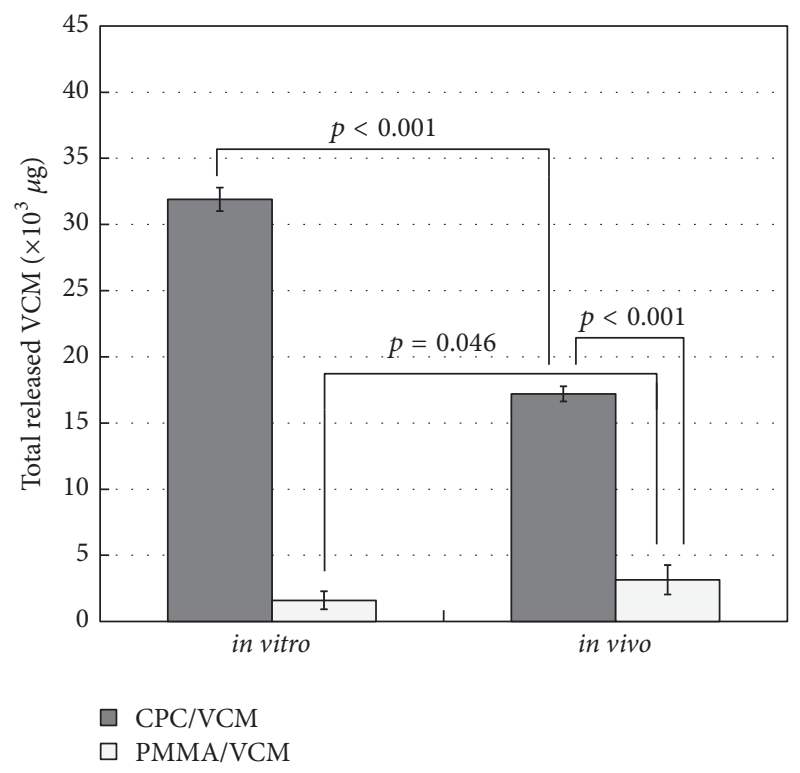

FIGURE 5: Total amount of VCM released from CPC/VCM and PMMA/VCM into rat tissues in 56 days. In vivo data for day 56 are directly cited from Figure $3\left(W_{56}\right)$. In vitro data were calculated by summing values from the elution curves generated following the daily exchange of solvent. The difference between in vitro and in vivo values indicates that $\mathrm{CPC/VCM}$ has the potential to continue to release residual VCM after day 56 in vivo. The average value and standard deviation of 10 (in vivo) and 3 (in vitro) of each specimen are shown.

of VCM in vivo compared to in vitro (Figure 5). This suggests that PMMA/VCM may be partly phagocytosed by macrophages as a foreign body in vivo [18], which would cause the elution rate to increase, despite there being less fluid volume around the material. However, VCM release from PMMA/VCM in vivo almost completely stopped after day 28 (Figure 3). In the two-stage exchange procedure following infected total joint replacement, reconstruction surgery is generally performed 6-8 weeks (or 12 weeks or more depending on the case) after the initial debridement and implantation of the antibiotic-impregnated cement material [19-21]. Therefore, according to our findings, PMMA/VCM is unable to provide antibiotic release for the clinically required period, despite showing longer release duration than that reported in vitro.

The ability of CPC to maintain high antibiotic concentrations in a relatively large amount of solvent has mainly been confirmed by in vitro studies [4-7]. Additionally, however, there are many published reports on the treatment of periprosthetic infections using antibioticimpregnated CPC as a potential alternative to conventional PMMA cement in clinical settings [8-10]. In the present study, CPC/VCM released more VCM over a longer duration than PMMA/VCM in vivo and in vitro (Figures 2-5). The amount of VCM released in vivo was approximately half that released in vitro, which may be due to less fluid volume around the material in the former compared to latter condition (Figure 5). Additionally, VCM release from 


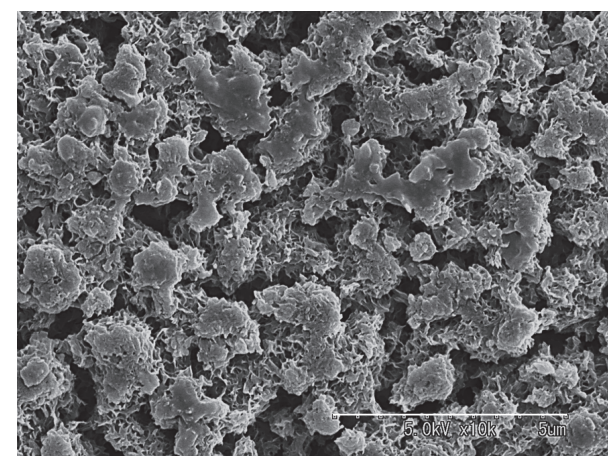

(a)

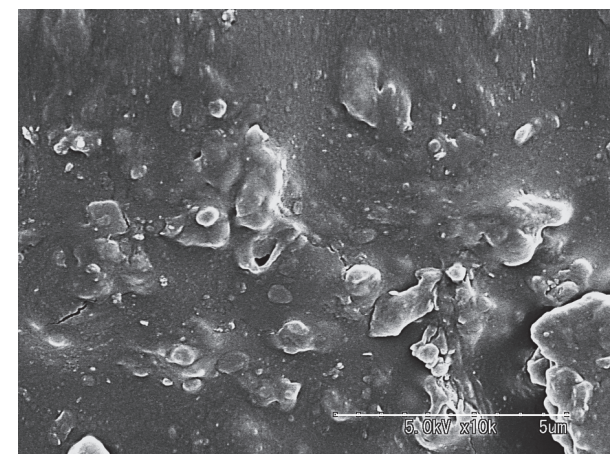

(b)

FIGURE 6: SEM images of the surfaces of CPC/VCM (a) and PMMA/VCM (b). Scale bars: $5 \mu \mathrm{m}$.

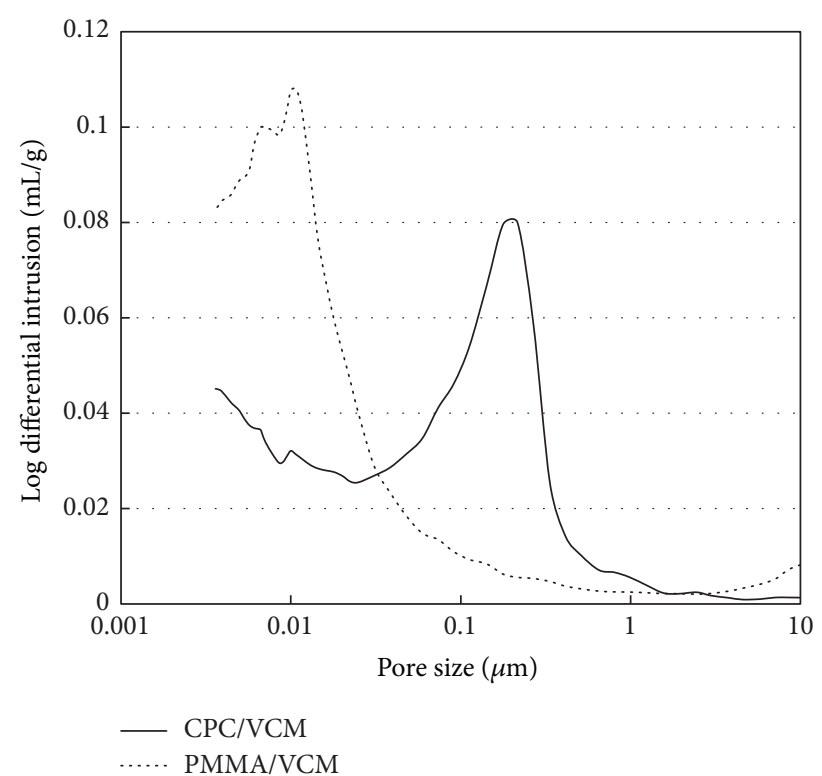

FIGURE 7: Mercury porosimetry analysis for CPC/VCM and PMMA/VCM. Mercury porosimetry plot of the pore sizes in CPC/VCM and PMMA/VCM. CPC/VCM and PMMA/VCM had pore sizes in the range of $0.025-0.65 \mu \mathrm{m}$ and $0.0036-0.074 \mu \mathrm{m}$, respectively.

CPC/VCM in vivo may continue after day 56 (Figure 3), with the approximate curve predicting release for the next 18 days (total 74 days). Given that the amount released per day exceeds the MIC $(1.56 \mu \mathrm{g})$ for more than 10 weeks after the initial implantation, our calculations suggest that CPC/VCM may provide sufficient antibacterial effect for the clinically required period. Therefore, CPC/VCM, which can provide antibiotic release for more than 8 weeks, may be a suitable material for providing local antibiotics to combat established postoperative infections.

Reports have indicated that an antibiotic release profile depends on the pore size of the cement material [12, 22]. The pore size of CPC impregnated with VCM and gentamicin is smaller than that for CPC impregnated with VCM alone, with the former showing decreased antibiotic elution compared to the latter [12]. In the present study, CPC/VCM had 20-fold larger pore size compared to PMMA/VCM (Figures 6 and 7). Therefore, the larger pore size of CPC/VCM may underlie its good release profile in vivo.

We observed long term release of VCM from CPC/VCM in vivo. However, long term use of CPC/VCM may be associated with problems such as resistance among staphylococci, including vancomycin-intermediate Staphylococcus aureus (VISA) [23], heteroresistant vancomycinintermediate Staphylococcus aureus strains (hVISA) [24], and vancomycin-resistant Staphylococcus aureus (VRSA) [25], and cement stability in clinical use. Further investigations are needed to determine the efficacy and safety of this treatment for established postoperative infections.

\section{Conclusions}

CPC/VCM showed longer retention time than PMMA/VCM in vitro and in vivo. The properties of CPC/VCM suggest that it is a promising agent for the treatment of established postoperative infections in clinical settings.

\section{Data Availability}

All data generated or analyzed during this study are included in this published article.

\section{Conflicts of Interest}

The authors declare that there are no conflicts of interest regarding the publication of this article.

\section{References}

[1] I. Scott, I. Stockley, and C. Getty, "Exchange arthroplasty for infected knee replacements. A new two-stage method," The Journal of Bone \& Joint Surgery (British Volume), vol. 75-B, no. 1, pp. 28-31, 1993.

[2] M. Cruciani, G. Gatti, L. Lazzarini et al., "Penetration of vancomycin into human lung tissue," Journal of Antimicrobial Chemotherapy, vol. 38, no. 5, pp. 865-869, 1996. 
[3] R. P. Pitto and I. A. Spika, "Antibiotic-loaded bone cement spacers in two-stage management of infected total knee arthroplasty," International Orthopaedics, vol. 28, no. 3, pp. 129-133, 2004.

[4] M. T. Ethell, R. A. Bennett, M. P. Brown, K. Merritt, J. S. Davidson, and T. Tran, "In vitro elution of gentamicin, amikacin, and ceftiofur from polymethylmethacrylate and hydroxyapatite cement," Veterinary Surgery, vol. 29, no. 5, pp. 375-382, 2000.

[5] O. Kisanuki, H. Yajima, T. Umeda, and Y. Takakura, "Experimental study of calcium phosphate cement impregnated with dideoxy-kanamycin B," Journal of Orthopaedic Science, vol. 12, no. 3, pp. 281-288, 2007.

[6] T. Sasaki, Y. Ishibashi, H. Katano, A. Nagumo, and S. Toh, "In vitro elution of vancomycin from calcium phosphate cement," The Journal of Arthroplasty, vol. 20, no. 8, pp. 1055-1059, 2005.

[7] K. Urabe, K. Naruse, H. Hattori et al., "In vitro comparison of elution characteristics of vancomycin from calcium phosphate cement and polymethylmethacrylate," Journal of Orthopaedic Science, vol. 14, no. 6, pp. 784-793, 2009.

[8] J. Aikawa, K. Urabe, M. Fujita et al., "Treatment of infected total knee arthroplasty by a local drug-delivery system with retention of the metal components," Kitasato Medical Journal, vol. 41, pp. 173-183, 2011.

[9] T. Iwakura, S. Y. Lee, T. Niikura et al., "Gentamycinimpregnated calcium phosphate cement for calcaneal osteomyelitis: A case report," Journal of Orthopaedic Surgery, vol. 22, no. 3, pp. 437-439, 2014.

[10] K. Uchiyama, N. Takahira, K. Fukushima et al., "Two-stage revision total hip arthroplasty for periprosthetic infections using antibiotic-impregnated cement spacers of various types and materials," The Scientific World Journal, vol. 2013, Article ID 147248, 7 pages, 2013.

[11] K. Sugo, K. Uchida, K. Naruse et al., "Elution mechanism of vancomycin and gentamicin from calcium phosphate cement," Phosphorus Research Bulletin, vol. 32, pp. 1-4, 2016.

[12] M. Uchino, K. Sugo, K. Naruse et al., "Elution Characteristics of Vancomycin, Gentamicin, and Vancomycin/Gentamicin Combination from Calcium Phosphate Cement," Advances in Orthopedic Surgery, vol. 2015, Article ID 257925, 5 pages, 2015.

[13] T. Watanabe, K. Ohashi, K. Matsui, and T. Kubota, "Comparative studies of the bactericidal, morphological and postantibiotic effects of arbekacin and vancomycin against methicillinresistant Staphylococcus aureus," Journal of Antimicrobial Chemotherapy, vol. 39, no. 4, pp. 471-476, 1997.

[14] K. Sugo, R. Kawashima, M. Nakasu, and T. Nakajima, "Antibiotic elution profile and physical properties of a novel calcium phosphate cement material," Journal of the Ceramic Society of Japan, vol. 124, no. 9, pp. 954-958, 2016.

[15] K. Anagnostakos, J. Kelm, T. Regitz, E. Schmitt, and W. Jung, "In vitro evaluation of antibiotic release from and bacteria growth inhibition by antibiotic-loaded acrylic bone cement spacers," Journal of Biomedical Materials Research Part B: Applied Biomaterials, vol. 72, no. 2, pp. 373-378, 2005.

[16] K. W. Klemm, "Antibiotic Bead Chains," Clinical Orthopaedics and Related Research, no. 295, pp. 63-76, 1993.

[17] J. Kelm, T. Regitz, E. Schmitt, W. Jung, and K. Anagnostakos, "In vivo and in vitro studies of antibiotic release from and bacterial growth inhibition by antibiotic-impregnated polymethylmethacrylate hip spacers," Antimicrobial Agents and Chemotherapy, vol. 50, no. 1, pp. 332-335, 2006.

[18] A. Sabokbar, Y. Fujikawa, D. W. Murray, and N. A. Athanasou, "Bisphosphonates in bone cement inhibit PMMA particle induced bone resorption," Annals of the Rheumatic Diseases, vol. 57, no. 10, pp. 614-618, 1998.

[19] T. Niikura, S. Y. Lee, T. Iwakura, Y. Sakai, R. Kuroda, and M. Kurosaka, "Antibiotic-impregnated calcium phosphate cement as part of a comprehensive treatment for patients with established orthopaedic infection," Journal of Orthopaedic Science, vol. 21, no. 4, pp. 539-545, 2016.

[20] B. A. Masri, C. P. Duncan, and C. P. Beauchamp, "Longterm elution of antibiotics from bone-cement: an in vivo study using the prosthesis of antibiotic-loaded acrylic cement (PROSTALAC) system," The Journal of Arthroplasty, vol. 13, no. 3, pp. 331-338, 1998.

[21] A. A. Hofmann, T. Goldberg, A. M. Tanner, and S. M. Kurtin, "Treatment of infected total knee arthroplasty using an articulating spacer: 2- to 12-year experience," Clinical Orthopaedics and Related Research, no. 430, pp. 125-131, 2005.

[22] C. Canal, D. Pastorino, G. Mestres, P. Schuler, and M.-P. Ginebra, "Relevance of microstructure for the early antibiotic release of fresh and pre-set calcium phosphate cements," Acta Biomaterialia, vol. 9, no. 9, pp. 8403-8412, 2013.

[23] K. Hiramatsu, H. Hanaki, T. Ino, K. Yabuta, T. Oguri, and F. C. Tenover, "Methicillin-resistant Staphylococcus aureus clinical strain with reduced vancomycin susceptibility," Journal of Antimicrobial Chemotherapy, vol. 40, no. 1, pp. 135-136, 1997.

[24] C. Liu and H. F. Chambers, "Staphylococcus aureus with heterogeneous resistance to vancomycin: Epidemiology, clinical significance, and critical assessment of diagnostic methods," Antimicrobial Agents and Chemotherapy, vol. 47, no. 10, pp. 3040-3045, 2003.

[25] S. Chang, D. M. Sievert, J. C. Hageman et al., "Infection with vancomycin-resistant Staphylococcus aureus containing the vanA resistance gene," The New England Journal of Medicine, vol. 348, no. 14, pp. 1342-1347, 2003. 


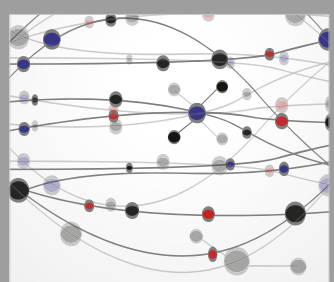

The Scientific World Journal
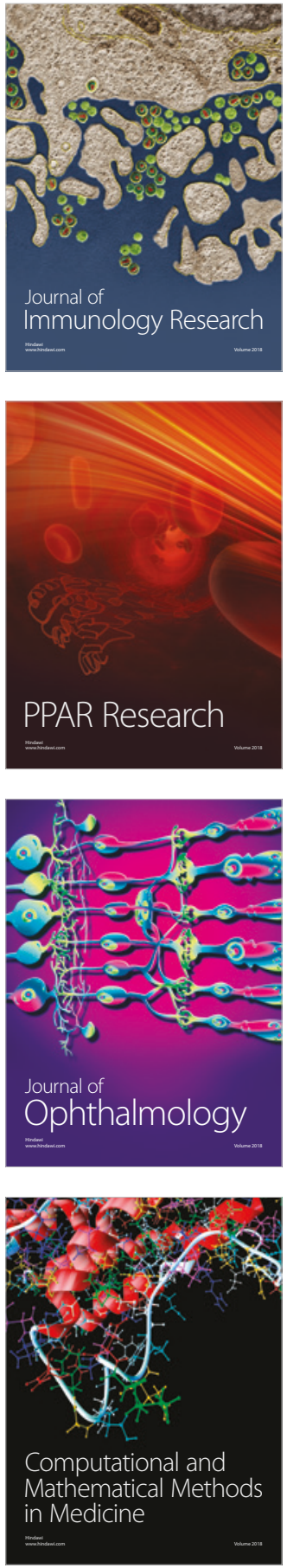

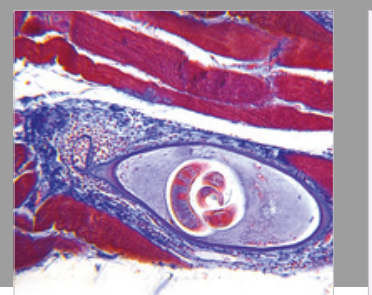

Gastroenterology Research and Practice

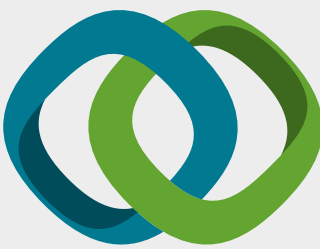

\section{Hindawi}

Submit your manuscripts at

www.hindawi.com
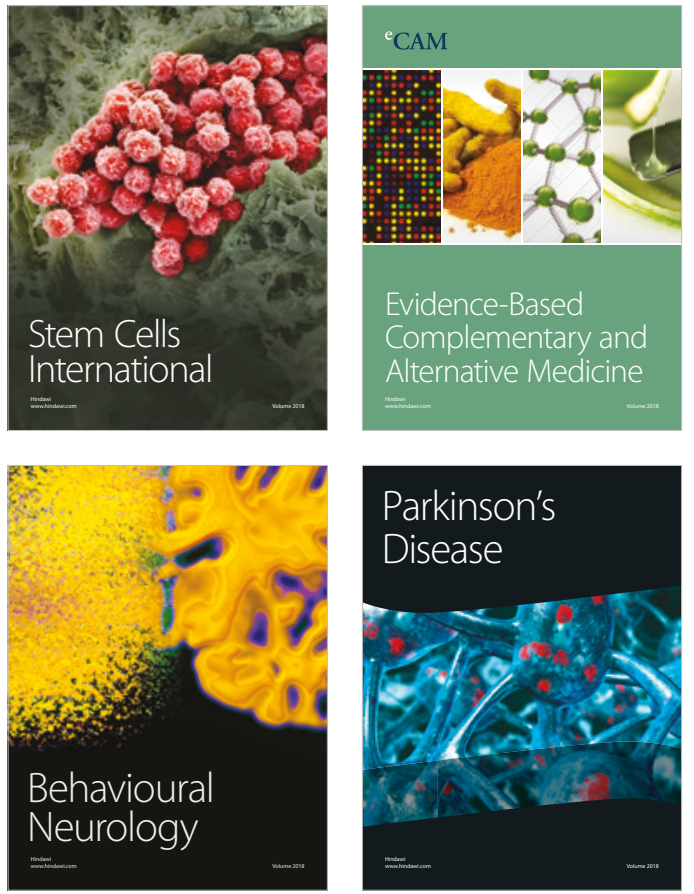

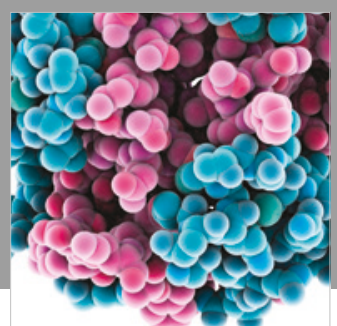

ournal of

Diabetes Research

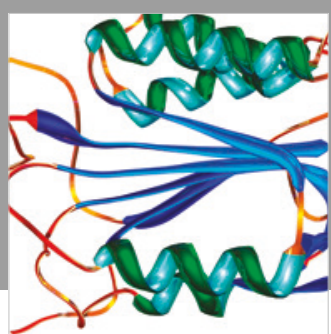

Disease Markers
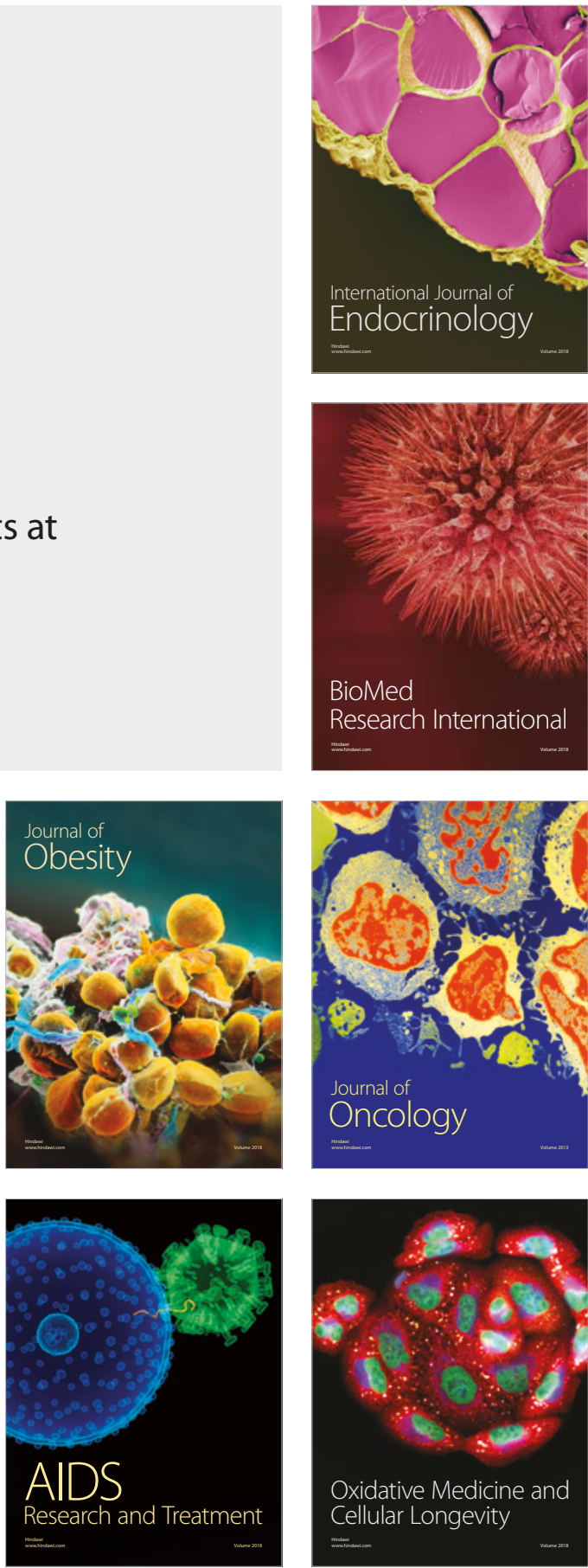